\title{
Solving Schrödinger Equation for Finite Potential Well Using the Iterative Method
}

\author{
Laith A. Al-Ani * and Russul K. Abid ** \\ Department of Physics, College of Science, Al-Nahrain University. \\ * Corresponding authors: laithalani2003@gmail.com \\ ** Corresponding authors: russulkhudir@gmail.com
}

\begin{abstract}
The quantum Finite Square Well (FSW) model is a well-known topic in most quantum mechanics (QM) books. A couple of equations can be derived from one dimensional Schrodinger equation for a finite potential well for describing the bound Eigen states within the well. Sometimes the FSW problem does not have an exact solution, yet, there are in fact exact solutions.

In this work a computational techniques is adopted to find the exact solution for FSW. To achieve this computational solution, a computer program has been written in Basic language for calculating the Eigen state energy for a particle confined in finite potential well using the iterative method (IM). Six values of potential width have been studied with potential depth. The results showed that wider potential width led us to more bound states, while narrower potential width led us to less bound states. Six values of potential depth have been studied with potential width. The results showed that larger potential depth led us to more bound states, while smaller potential width led us to less bound states.

A Comparison Between Finite and Infinite Potential Well has been also presented. The result of comparison showed that energy levels of an infinite well are much higher than that the corresponding energy levels for finite potential well.

In general, the matching between the results of the iterative method and the graphical method (GM) proving that the iteration method can be regarded as a useful tool for describing the solutions of the 1-dimensional FSW problem.

[DOI: 10.22401/ANJS.22.4.07]
\end{abstract}

Keywords: Finite Potential Well, Computational Techniques, Schrödinger Equation.

\section{Introduction}

The quantum mechanics mathematics is usually introduced through physical systems described by one dimensional wells $[1,2]$. These examples are used to show the emergence of quantized states as solutions of the Schrödinger equation subject to appropriate boundary conditions. Unfortunately, there are not many potentials for which the bound state energies can be expressed in closed form. The classic example is the infinite square well, but it is obviously artificial. In the more realistic case where the potential well is finite, the allowed energies as functions of the barrier height can be found numerically by solving a transcendental equation [3], by graphical methods [4- 6] or by various approximation techniques [7-10].

Quantum well models are essential for the structure of semiconductor devices, for example the quantum well infrared photodetector (QWIP), which is utilized for infrared imaging applications [11]. The QWIP relies upon a quantum well that has been sized so that the energy of an electron in the first excited state is quite near the threshold of confinement in the well.

The QWIP is therefore very sensitive to the arrival of a single photon. There are numerous different uses of quantum well models in nanostructures; the textbook by Harrison [12] give a good literature survey in this field.

The finite quantum well is of great practical importance because it forms the basis for understanding low-dimensional structures such as quantum well devices [11].

\section{2- Derivation of Transact Equations}

The choice of potential V (r) decides what system that will be modeled. A potential with an easy analytic solution can be used for testing the accuracy, convergence and stability of the numerical methods. A more advanced potential that cannot be solved analytically may be a better approximation of the real problem [13]. 
Consider the potential shown in Fig.(1), the particle has energy, $E$, less than $V_{o}$, and is bound to the well $[1,14]$.

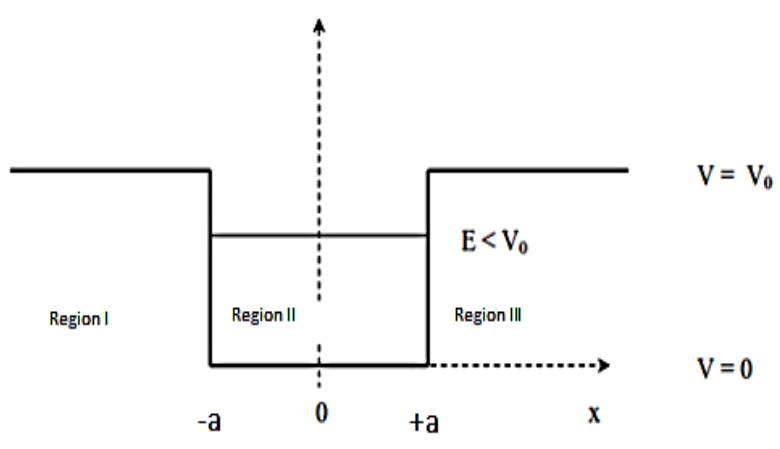

Fig.(1): A finite square well, depth, $V_{o}$, width $2 a$.

The finite $1 \mathrm{D}$ square well potential $\mathrm{V}(\mathrm{x})$ can be described by the following [14 ].

$V(x)=\left\{\begin{array}{ccc}V_{o} & \text { if } & x \leq-a \\ 0 & \text { if } & -a \leq x \leq a \\ V_{o} & \text { if } & x \geq-a\end{array}\right\}$

Consider the time-independent Schrodinger equation in one dimension,

$\frac{d^{2} \psi}{d x^{2}}+\frac{2 m}{\hbar^{2}}\left(E-V_{(x)}\right) \psi=0$

Fig.(1) divide the width into three regions I, II, and III according to the potential.

\section{Region I}

In this region $x \leq-a$ and $V_{(x)}=V_{o}$, then Schrodinger equation is written as:

$\frac{d^{2} \psi_{I}}{d x^{2}}+\frac{2 m}{\hbar^{2}}\left(E-V_{o}\right) \psi_{I}=0$

Let

$\beta^{2}=\frac{2 m}{\hbar^{2}}\left(V_{o}-E\right)$

Then

$\frac{d^{2} \psi_{I}}{d x^{2}}-\beta^{2} \psi_{I}=0$

The solutions to this differential equation are:

$\psi_{I}=C e^{\beta x}+D e^{-\beta x}$

But, since $\psi_{I} \rightarrow 0 \quad$ as $x \rightarrow-\infty$. This condition is satisfied when the constant $\mathrm{D}$ equal to 0 , then the solution became
$\psi_{I}=C e^{\beta x}$

\section{Region III}

In region III, Schrodinger equation is written as:

$\frac{d^{2} \psi_{I I I}}{d x^{2}}+\frac{2 m}{\hbar^{2}}\left(E-V_{o}\right) \psi_{I I I}=0$

Or

$\frac{d^{2} \psi_{I I I}}{d x^{2}}-\beta^{2} \psi_{I I I}=0$

$\psi_{\text {III }}=C e^{\beta x}+D e^{-\beta x}$

but since $\psi_{I I I} \rightarrow 0 \quad$ as $x \rightarrow+\infty$, This condition is satisfied when the constant $C$ $=0$, then the solution became

$\psi_{I I I}=D e^{-\beta x}$

\section{Region II}

In region II the potential equal to zero, then equation (2) can be written as

$-\frac{\hbar^{2}}{2 m} \frac{d^{2} \psi_{I I}}{d x^{2}}=E \psi_{I I}$

Or

$\frac{d^{2} \psi_{I I}}{d x^{2}}+\frac{2 m E}{\hbar^{2}} \psi_{I I}=0$

Let

$\alpha^{2}=\frac{2 m E}{\hbar^{2}}$

Then equation (12) became

$\frac{d^{2} \psi_{I I}}{d x^{2}}+\alpha^{2} \psi_{I I}=0$

and the solution [14].

$\psi_{I I}=A \operatorname{Sin} \alpha x+B \operatorname{Cos} \alpha x$

where A, B, C, D are constants

To find the constants values, four equations must be found. These equations can be found by applying the condition of continuity

$$
\begin{aligned}
& \left\{\begin{array}{c}
\psi_{I}=\psi_{I I} \\
\frac{d \psi_{I}}{d x}=\frac{d \psi_{I I}}{d x}
\end{array}\right\} \quad \text { at } \quad x=-a \\
& \left\{\begin{array}{c}
\psi_{I I I}=\psi_{I I} \\
\frac{d \psi_{I I I}}{d x}=\frac{d \psi_{I I}}{d x}
\end{array}\right\} \quad \text { at } \quad x=a
\end{aligned}
$$


Since the potential is symmetric, then the wave functions have defined parity (odd or even).

\section{Even parity}

To satisfy the even parity condition, the following conditions must be satisfied, $\psi_{(+x)}=\psi_{(-x)}$ and the value of $\mathrm{A}$ in equation (16) must be zero. Equation (16) can be re written as

$\psi_{I I}=B \operatorname{Cos} \alpha x$

Also, the constant $\mathrm{C}$ in equation (7) and the constant $\mathrm{D}$ in equation (11) must be equal then equations can be re written as

$\psi_{I}=C e^{\beta x}$

$\psi_{I I I}=C e^{-\beta x}$

By applying the condition of continuity at $\mathrm{x}=$ $+\mathrm{a}\left(\right.$ i.e. $\left.\psi_{I I}=\psi_{I I I}\right)$

$B \operatorname{Cos} \alpha a=C e^{-\beta a}$

and

$-\alpha B \operatorname{Sin} \alpha a=-\beta C e^{-\beta a}$

Dividing equation (21) on equation (20) yield

$\alpha \tan \alpha \mathrm{a}=\beta \quad$ for even parity

\section{odd parity}

In this case $\psi_{(+x)}=-\psi_{(-x)}$ and the value of $B$ in equation (16) must be zero. Equation (16) can be re written as

$\psi_{I I}=A \operatorname{Sin} \alpha x$

Also, the constant $\mathrm{C}$ in equation (7) equal to the negative value of constant $\mathrm{D}$ in equation (11) then equations can be re written as

$\psi_{I}=-D e^{\beta x}$

$\psi_{I I I}=D e^{-\beta x}$

By applying the condition of continuity at $\mathrm{x}=$ $+\mathrm{a}\left(\right.$ I.e. $\left.\psi_{I I}=\psi_{I I I}\right)$

$A \operatorname{Sin} \alpha a=D e^{-\beta a}$

And

$\alpha A \operatorname{Cos} \alpha a=-\beta D e^{-\beta a}$ dividing equation (27) by equation (26) to eliminate $\mathrm{A}$ and $\mathrm{D}$ gives

$\alpha \cot \alpha \mathrm{a}=-\beta \quad$ for odd parity

now let

$k=\alpha a$

by using equation (14).

$k=\frac{a}{\hbar} \sqrt{2 m E}$

or

$E=\frac{\mathrm{k}^{2} \hbar^{2}}{2 \mathrm{~m} \mathrm{a}^{2}}$

and let

$b=\frac{a}{\hbar} \sqrt{2 \mathrm{mV}_{\mathrm{o}}}$

From equation (3) and equation (14)

$\frac{\alpha}{\beta}==\sqrt{\frac{E}{\left(V_{o}-E\right)}}=\sqrt{\frac{k^{2}}{\left(b^{2}-k^{2}\right)}}$

Also from equation (22) for even parity and equation(28) for odd parity the following equations can be extracted

$\frac{\alpha}{\beta}=\operatorname{Cot} \alpha \mathrm{a} \quad$ for even parity

$\frac{\alpha}{\beta}=-\tan \alpha$ a for odd parity

Note that: since $\beta=\sqrt{\frac{2 m}{\hbar^{2}}\left(V_{o}-E\right)}$ and $\alpha=\sqrt{\frac{2 \mathrm{mE}}{\hbar^{2}}}$, in both equation (4) and (14) respectively, there is only one unknown, the energy, $E$; so we should be able to solve for the energy.

The solution of these implicit equations can be obtained by using either the graphical solution method or using suitable numerical method like Iterative method, NewtonRaphson method ....etc.

\section{3- Result and Discussions}

A computer program was written in basic language is utilized to calculate the Eigen value energies for a particle confined in finite and infinite potential well by adopting iterative method. The main program code was built with a number of supplement sub programs (routines). 
Fig.(2) show the flow chart of the main program which is used for calculating the Eigen values using the iterative method.

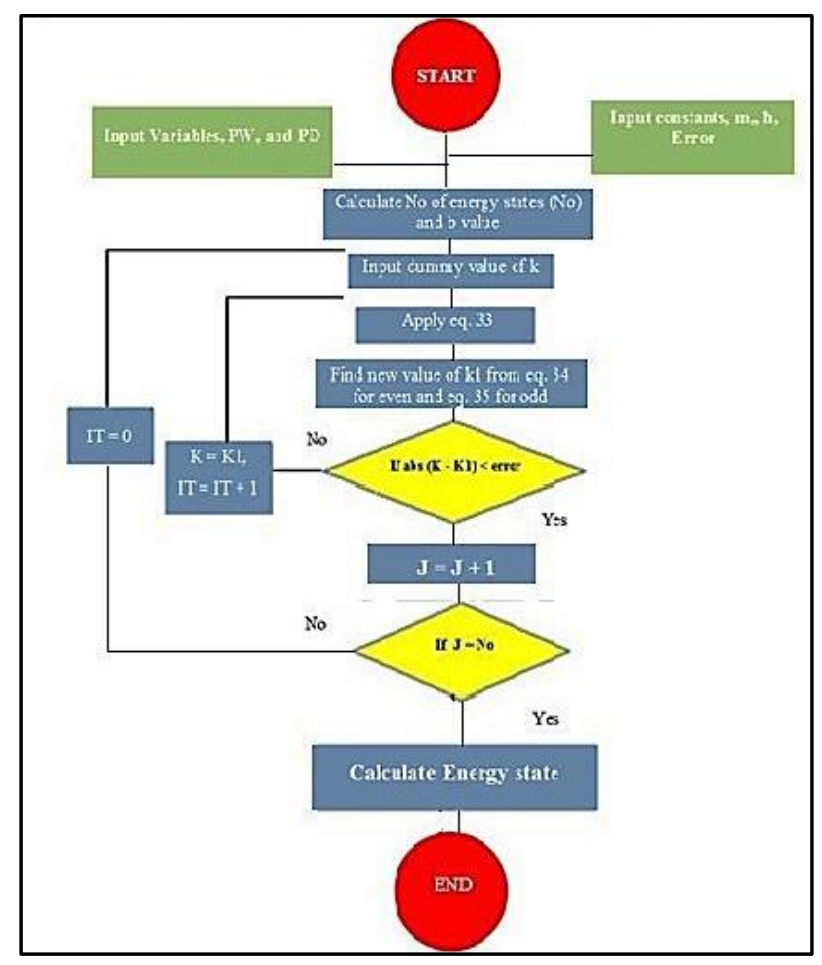

\section{Fig.(2): Flow chart utilized for calculating} the Eigen values energies.

This program responsible on the process of the iterative method by utilizing equations (33) with eq (34) for even parity, and eq (35) for odd parity and continue looping between these equations until the result became less or equal the input of the tolerance error. The first step in the main program start by determining the number of energy states according to the input values of potential depth (PD) and potential width (PW).

To test the output results precision throughout the execution the program, a comparison study is made between the results obtained from graphical method (GM) and the results obtained from the adopted numerical iterative method (IM). The Finite Square Well program is performed to calculate the EigenEnergies with a tolerance accuracy of 0.000001 (This value can be altered manually in the code, by changing the constant tolerance value).

Fig.(3) show a graphical solution for the energy Eigenvalues of the six bound states for an electron confined in a $4 \mathrm{~A}^{\circ}, 75 \mathrm{eV}$ finite potential well [15].

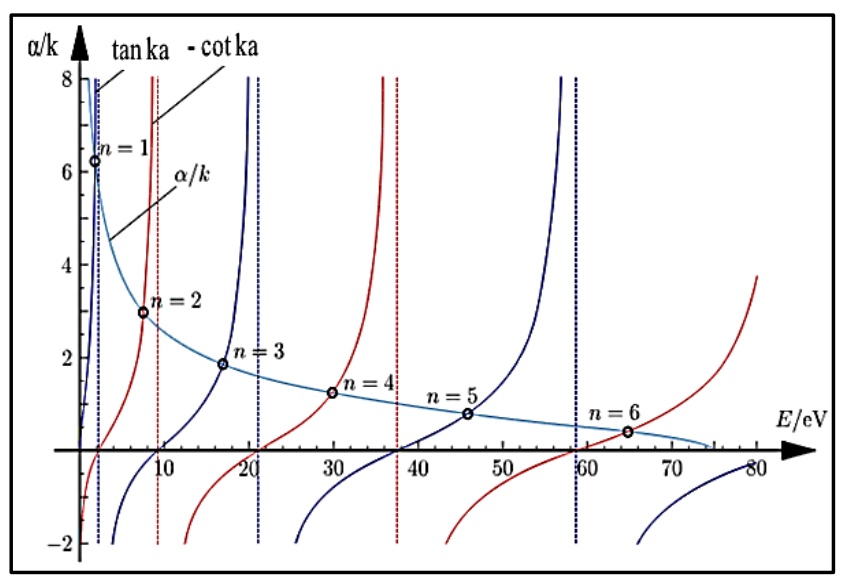

Fig.(3): A graphical solution for the energy

Eigenvalues of the six bound states of an electron in a $4 A^{o}$, 75 eV finite potential well [16].

Table (1) present the Eigen value energy results between the result obtained from graphical for potential width $4 \mathrm{~A}^{\circ}$ and potential depth $75 \mathrm{eV}$ [15] in addition to the obtained result from the iterative method (IM) of the present work for the same boundary condition.

Table (1)

Eigen value from GM and IM for PW $4 A^{\circ}$ and PD $75 \mathrm{eV}$.

\begin{tabular}{|l||c||c||c||}
\hline$n$ & Parity & $\begin{array}{c}\text { Eigen value } \\
(\mathrm{eV})(\mathrm{GM}) \\
\mathrm{Ref}[15]\end{array}$ & $\begin{array}{c}\text { Eigen Value } \\
(\mathrm{eV})(\mathrm{IM}) \\
\text { Present Work }\end{array}$ \\
\hline 1 & Even & 1.9 & 1.901826 \\
\hline 2 & Odd & 7.6 & 7.586675 \\
\hline 3 & Even & 16.9 & 16.95550 \\
\hline 4 & Odd & 29.9 & 29.95245 \\
\hline 5 & Even & 46.1 & 46.17400 \\
\hline 6 & Odd & 64.6 & 64.75384 \\
\hline
\end{tabular}

\section{3-1 Potential Width (PW) Effect}

Four values of potential width $(2,4,6$, and $\left.8 \mathrm{~A}^{\circ}\right)$ are studied with potential depth $(16 \mathrm{eV})$. The result of energy Eigen values for these boundary condition parameters using the iteration method are tabulated in table (2) . 
Table (2)

The result of Eigen value from numerical method for four values of potential width and potential depth $16 \mathrm{eV}$.

\begin{tabular}{|c|c|c|c|c|}
\hline $\mathrm{n}$ & $\begin{array}{l}\text { PD } \\
(\mathrm{eV}) \\
\end{array}$ & $\begin{array}{l}\mathrm{PW} \\
\left(\mathrm{A}^{\mathrm{o}}\right)\end{array}$ & $\begin{array}{c}\mathrm{n}^{\text {th }} \\
\text { Result }\end{array}$ & $\begin{array}{c}\text { Eigen Value } \\
(\mathrm{eV})(\mathrm{IM})\end{array}$ \\
\hline 1 & & 2 & 2 & $\begin{array}{l}4.137227 \\
15.39703\end{array}$ \\
\hline 2 & & 4 & 3 & $\begin{array}{l}1.513965 \\
5.919476 \\
12.55995\end{array}$ \\
\hline 3 & 16 & 6 & 4 & $\begin{array}{c}0.7726148 \\
3.067378 \\
6.798559 \\
11.72331\end{array}$ \\
\hline 4 & & 8 & 6 & $\begin{array}{c}0.466942 \\
1.861466 \\
4.162131 \\
7.321645 \\
11.22829 \\
15.4325\end{array}$ \\
\hline
\end{tabular}

The results of table (2) show that larger potential width led us to more bound state, while smaller potential width led us to less bound states

\section{3-2 Potential Depth (PD) Effect}

Six values of potential depth $(4,8,12,16$, 20 and $24(\mathrm{eV})$ is studied with potential width $4\left(\mathrm{~A}^{\mathrm{o}}\right)$. The result of energy Eigen values for these boundary condition parameters using the iteration method are tabulated in Table (3). The results of table (3) show that the following remarks:

- larger PD led us to more bound states, while smaller PD led us to less bound states

- The energy of the $n^{\text {th }}$ state is less than the infinite potential well. This means inside finite potential well type of potentials the number of quantum states is finite
Table (3)

The result of Eigen value from numerical method for six values of potential depth $(e V)$ and potential width $4 A^{\circ}$.

\begin{tabular}{|c|c|c|c|c|}
\hline $\mathrm{n}$ & $\begin{array}{l}\text { PW } \\
\left(A^{\circ}\right)\end{array}$ & $\begin{array}{l}\text { PD } \\
(\mathrm{eV})\end{array}$ & $\begin{array}{c}\mathrm{n}^{\text {th }} \\
\text { Result }\end{array}$ & $\begin{array}{c}\text { Eigen Value } \\
(\mathrm{eV})(\mathrm{IM})\end{array}$ \\
\hline 1 & \multirow{6}{*}{4} & 4 & $\begin{array}{l}1 \\
2\end{array}$ & $\begin{array}{l}1.034307 \\
3.849257\end{array}$ \\
\hline 2 & & 8 & $\begin{array}{l}1 \\
2\end{array}$ & $\begin{array}{l}1.285277 \\
4.846983\end{array}$ \\
\hline 3 & & 12 & $\begin{array}{l}1 \\
2 \\
3\end{array}$ & $\begin{array}{l}1.423374 \\
5.504372 \\
12.28175\end{array}$ \\
\hline 4 & & 16 & $\begin{array}{l}1 \\
2 \\
3\end{array}$ & $\begin{array}{l}1.513965 \\
5.919476 \\
12.55995\end{array}$ \\
\hline 5 & & 20 & $\begin{array}{l}1 \\
2 \\
3\end{array}$ & $\begin{array}{l}1.582808 \\
6.225575 \\
13.46658\end{array}$ \\
\hline 6 & & 24 & $\begin{array}{l}1 \\
2 \\
3 \\
4\end{array}$ & $\begin{array}{c}1.633098 \\
6.44743 \\
14.09409 \\
24.33458\end{array}$ \\
\hline
\end{tabular}

\section{3-Comparison Between Finite and Infinite Potential Well}

Table (4) presents the comparison for the four bound stated in a $2 \mathrm{~nm}\left(20 \mathrm{~A}^{\circ}\right)$ potential width, and $1 \mathrm{eV}$ potential depth for one dimensional finite potential well and infinite potential well

Table (4)

Comparison of infinite and Finite Potential Wells.

\begin{tabular}{|c|c|c|c|c|}
\hline \multirow{3}{*}{$\begin{array}{l}\text { PW } \\
\left(A^{0}\right)\end{array}$} & \multirow{3}{*}{$n^{\text {th }}$} & \multicolumn{3}{|c|}{ Eigen Value (eV) } \\
\hline & & \multirow{2}{*}{$\begin{array}{c}\begin{array}{c}\text { Ref } \\
{[16]}\end{array} \\
\text { Infinite } \\
\text { Potential }\end{array}$} & \multicolumn{2}{|c|}{ Present Work } \\
\hline & & & $\begin{array}{l}\text { Infinite } \\
\text { Potential }\end{array}$ & $\begin{array}{c}\text { Finite } \\
\text { Potential } \\
(1) \mathrm{eV}) \\
\end{array}$ \\
\hline \multirow{4}{*}{20} & 1 & 0.094 & 0.094138 & 0.065849 \\
\hline & 2 & 0.377 & 0.376551 & 0.260150 \\
\hline & 3 & 0.848 & 0.847239 & 0.569726 \\
\hline & 4 & -------- & 1.506203 & 0.941164 \\
\hline
\end{tabular}

Fig.(4) showes The four bound stated in a $2 \mathrm{~nm}\left(20 \mathrm{~A}^{\mathrm{o}}\right)$ potential width, and $1 \mathrm{eV}(\mathrm{PD})$ 
for one dimensional a- finite potential well $b$ infinite potential well.

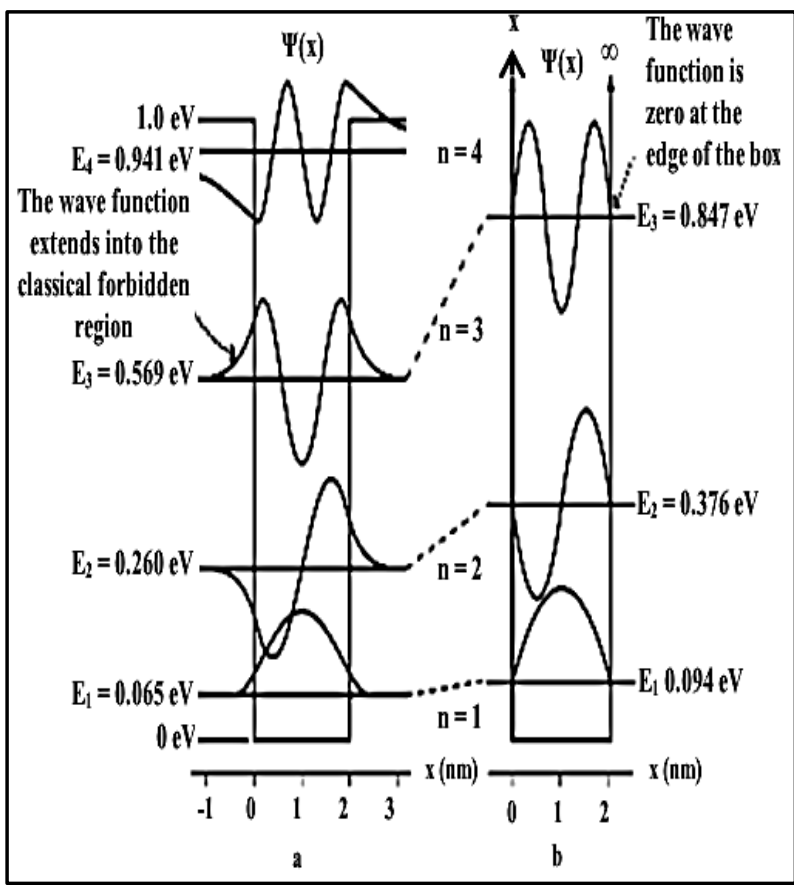

Fig.(4): The four bound stated in a $2 \mathrm{~nm}$ $\left(20 \mathrm{~A}^{\circ}\right)$ potential width, and $1 \mathrm{eV}(\mathrm{PD})$ for one dimensional a-finite potential well $b$ infinite potential well.

Table (4) and Fig.(4) showes that the calculated energy levels in the finite potential well are lower than the corresponding energy levels in the infinite potential well of the same width $(\mathrm{E} 1=0.094 \mathrm{eV}, \mathrm{E} 20.377 \mathrm{eV}$, and E3 = $0.848 \mathrm{eV})$. This is not surprising because the wave function in the finite potential well extends into the classically forbidden region, according to that the corresponding wavelengths are longer than those in the infinite potential well and therefore reduces its kinetic energy.

Table (5) presents the results of Eigen states values for finite potential well for potential width $4 \mathrm{~A}^{\mathrm{o}}$ with three values of potential depth $(25,50$, and $125 \mathrm{eV})$ in addition to the corresponding calculated Eigen states values for infinite potential well. The results also show that how the corresponding energy levels of an infinite well are much higher. In finite potential well the particle is not strictly contained and the location extends into classically forbidden region.

Table (5) also presents the percentage error between the calculated Eigen states values for finite potential well and the corresponding calculated Eigen states values for infinite potential well. It's clear from this results that percentage error decreased when the potential depth increased.

Table (5)

Comparison of infinite and Finite Potential Wells.

\begin{tabular}{|c|c|c|c|c|c|}
\hline \multirow{3}{*}{$\begin{array}{l}\mathrm{PW} \\
\left(\mathrm{A}^{\circ}\right)\end{array}$} & \multirow{3}{*}{$\mathrm{n}^{\text {th }}$} & \multicolumn{4}{|c|}{ Eigen Value (eV) } \\
\hline & & \multicolumn{4}{|c|}{ Present Work } \\
\hline & & $\begin{array}{c}\text { Infinite } \\
\text { Potential } \\
\end{array}$ & $\begin{array}{l}\text { PD } \\
(\mathrm{eV}) \\
\end{array}$ & $\begin{array}{c}\text { finite } \\
\text { Potential } \\
\end{array}$ & Error\% \\
\hline \multirow{17}{*}{4} & 1 & 2.35344 & \multirow{4}{*}{25} & 1.64623 & 30.1 \\
\hline & 2 & 9.41376 & & 6.503752 & 30.9 \\
\hline & 3 & 21.18097 & & 14.24314 & 32.8 \\
\hline & 4 & 37.65506 & & 23.5291 & 37.5 \\
\hline & 1 & 2.35344 & \multirow{5}{*}{50} & 1.81439 & 22.9 \\
\hline & 2 & 9.41376 & & 7.222725 & 23.3 \\
\hline & 3 & 21.18097 & & 16.10431 & 23.9 \\
\hline & 4 & 37.65506 & & 28.16892 & 25.2 \\
\hline & 5 & 58.83604 & & 42.5557 & 27.7 \\
\hline & 1 & 2.35344 & \multirow{8}{*}{125} & 1.99215 & 15.4 \\
\hline & 2 & 9.41376 & & 7.95804 & 15.5 \\
\hline & 3 & 21.18097 & & 17.86378 & 15.6 \\
\hline & 4 & 37.65506 & & 31.64409 & 16.0 \\
\hline & 5 & 58.83604 & & 49.18309 & 16.4 \\
\hline & 6 & 84.72389 & & 70.26498 & 17.0 \\
\hline & 7 & 115.3186 & & 94.41186 & 18.1 \\
\hline & 8 & 150.6203 & & 119.8218 & 20.4 \\
\hline
\end{tabular}

\section{Conclusions}

- The iterative method was succeed to solve the Schrodinger equation for finite potential well. This method is very accurate and efficient.

- More bound state can be found with larger potential width, while smaller potential width led us to less bound states

- More bound states can be found with larger potential depth while smaller potential depth led us to less bound states

- The number of energy of the $\mathrm{n}^{\text {th }}$ state is less than the infinite potential well. This means inside finite potential well type of potentials the number of quantum states is finite

- The percentage error decreased between the calculated Eigen states values for finite potential well and the corresponding calculated Eigen states values for infinite potential well when the potential depth increased. 


\section{References}

[1] D. J. Griffiths, "Introduction to Quantum Mechanics", Prentice Hall, Englewood Cliffs, NJ, 2005.

[2] R. A. Serway, C. J. Moses, and C. A. Moyer, "Modern Physics", Brooks Cole, Belmont, MA, 2005.

[3] R. D. Murphy, and J. M. Phillips, "Boundstate eigenvalues of the square potential", Am. J. Phys. 44, 574-576, 1976.

[4] P. H. Pitkanen, "Rectangular potential well in quantum mechanics", Am. J. Phys. 23, 111-113, 1955.

[5] P. G. Guest, "Graphical solutions for the square well”, Am. J. Phys. 40, 1175-1176 1972.

[6] J. V. Mallow, "Simple graphical solution for the finite square well with no change of variables", Am. J. Phys. 64, 1072-1073, 1996.

[7] D. J. Searle's, and E. I. von NagyFelsobuki, "Numerical experiments in quantum physics: Finite-element method," Am. J. Phys. 56, 444-448, 1988.

[8] S. Garrett, "Bound state energies of a particle in a finite square well: A simple approximation," Am. J. Phys. 47, 195-196, 1979.

[9] J.-F. Bloch, "A new approach to bound states in potential wells," Am. J. Phys. 69, 1177-1181, 2001.

[10] O. F. de Alcantara Bonfim and D. J. Griffiths, "Exact and approximate energy spectrum for the finite square well and related potentials", Am. J. Phys., Vol. 74, No. 1, January 2006.

[11] H. Schneider, and H.C. Liu. Quantum well infrared photodetectors: physics and applications. Springer. 2007.

[12] P. Harrison. "Quantum wells, wires and dots: theoretical and computational physics of semiconductor nanostructures", 3rd ed. Wiley. 2009.

[13] V. Lina, A. Louise, and M. Jonas, "Numerical approaches to solving the time-dependent Schrodinger equation with different potentials", M.Sc. thesis, Uppsala University, 2016.

[14] B.H. Bransden and C.J. Joachain, Quantum Mechanics, Pearson/Prentice Hall, 2000.
[15] Website: https://en.wikibooks.org/wiki/Materials_in _Electronics/Confined_Particles/1D_Finite Wells

[16] Website: http://www.colorado.edu/physics/phys2170/ 